SUPPLEMENTARY MATERIALS

\title{
In Vitro Biocompatibility of Decellularized Cultured Plant Cell-Derived Matrices
}

Nhi V. Phan ${ }^{1}$, Tristen Wright ${ }^{2}$, M. Masrur Rahman ${ }^{1}$, Jianfeng $\mathrm{Xu}^{3,4}$, Jeannine M. Coburn ${ }^{1, *}$

${ }^{1}$ Department of Biomedical Engineering, Worcester Polytechnic Institute, Worcester, MA, ${ }^{2}$ Department of Biological Science, Arkansas State University, Jonesboro, AR, ${ }^{3}$ Arkansas Biosciences Institute, Arkansas State University, Jonesboro, AR

${ }^{4}$ College of Agriculture, Arkansas State University, Jonesboro, AR 

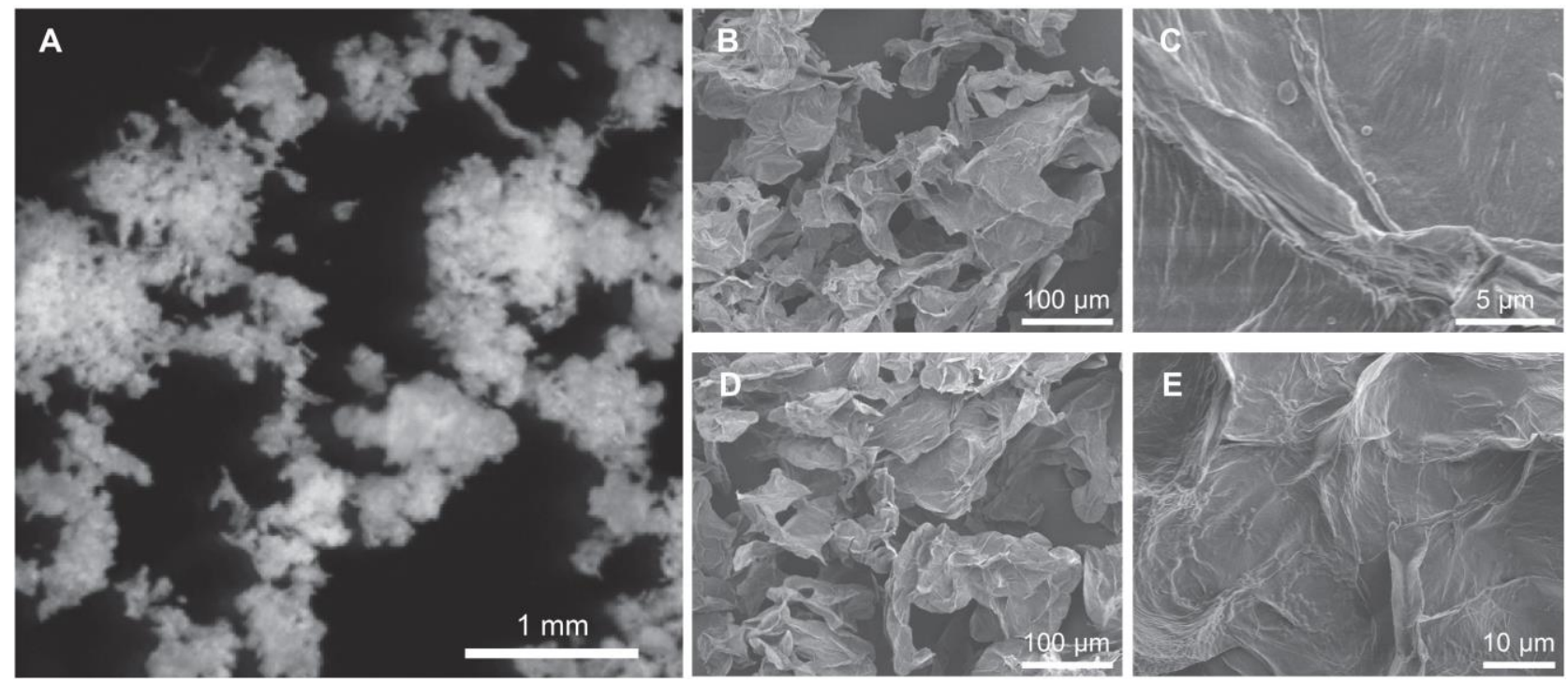

Figure S1. Morphology of BY-2 cell-derived matrices post-lyophilization and treatment. (A) Lyophilized BY-2 cell-derived matrices exhibited a powdery appearance. (B and C) SEM image of DNase treated BY-2 cell-derived matrices at (B) low and (C) high magnification. (D and E) SEM image of buffer-only treated BY-2 cell-derived matrices at (D) low and (E) high magnification. 

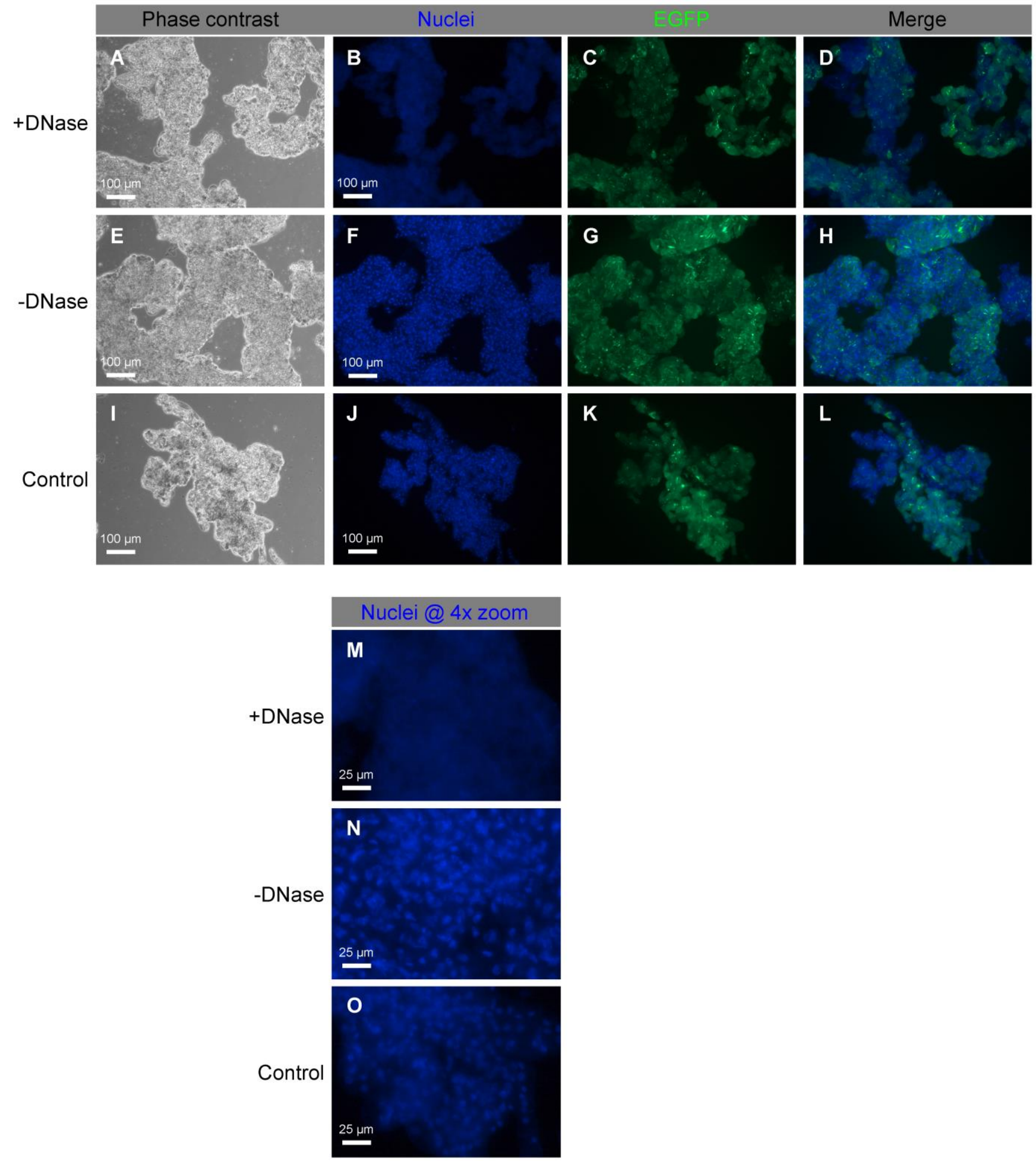

Figure S2. Fluorescent and phase contrast microscopy images of the processed BY -2 cell-derived matrices. Images of BY-2 cell-derived matrices after treatment with (A-D, M) $1 \mathrm{mg} / \mathrm{mL}$ DNase (+DNase) or (E-H, N) buffer-only (-DNase) for $30 \mathrm{~min}$ at $37^{\circ} \mathrm{C}$. (I-L, O) Images of as-lyophilized BY-2 cells. (B, $\mathbf{F}, \mathbf{J}, \mathbf{M}, \mathbf{N}, \mathbf{O})$ The nuclei of the + DNase treatment group were disrupted as evidenced by loss of focal staining as compared to the -DNase and control groups. $(\mathbf{C}, \mathbf{G}, \mathbf{K})$ Qualitatively, EGFP was retained within all matrices throughout the processing as evidenced by fluorescence in the green channel. BY -2 cells were proliferated in MS medium. All brightfield and fluorescent images in A-L were taken using the same magnification. 


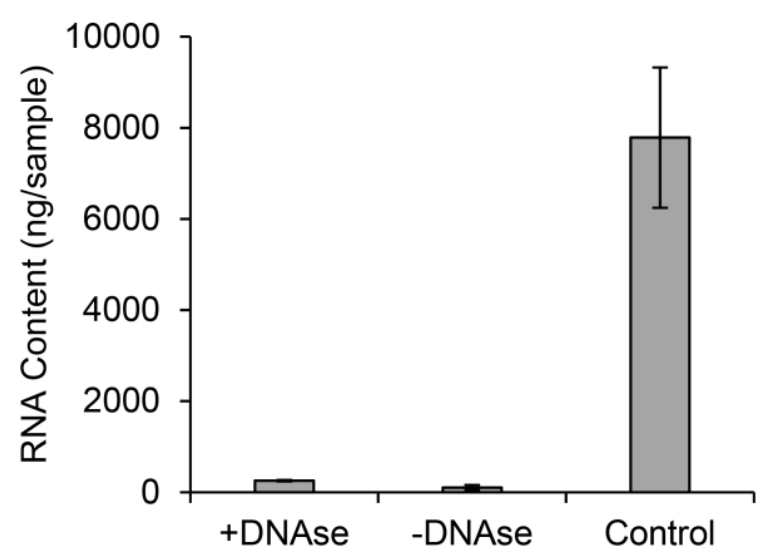

Figure S3. RNA quantification of the decellularized BY-2 cell-derived matrices. RNA content of samples treated with DNase (+DNase) or buffer-only (-DNase) for $30 \mathrm{~min}$ at $37^{\circ} \mathrm{C}$ or as-lyophilized controls. Data are presented as the mean \pm standard deviation of three samples. 

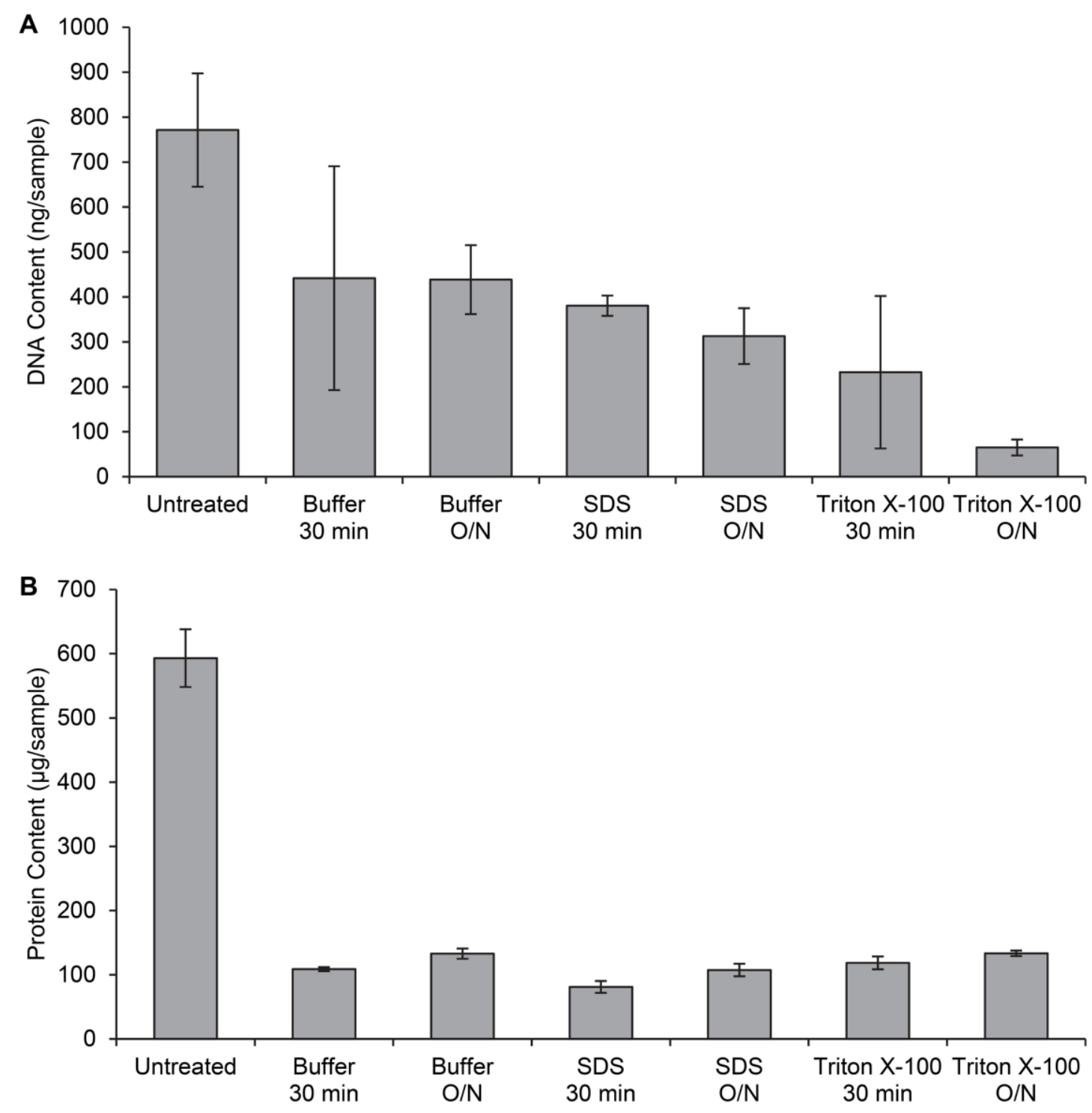

Figure S4. Effect of treating lyophilized BY -2 cells with surfactants. The lyophilized BY-2 cells were treated $0.25 \%$ SDS or $0.1 \%$ Triton X-100 for 30 minutes or overnight. (A) DNA and (B) protein content of the resulting matrices. Data are presented as mean \pm standard deviation from three independent samples. 


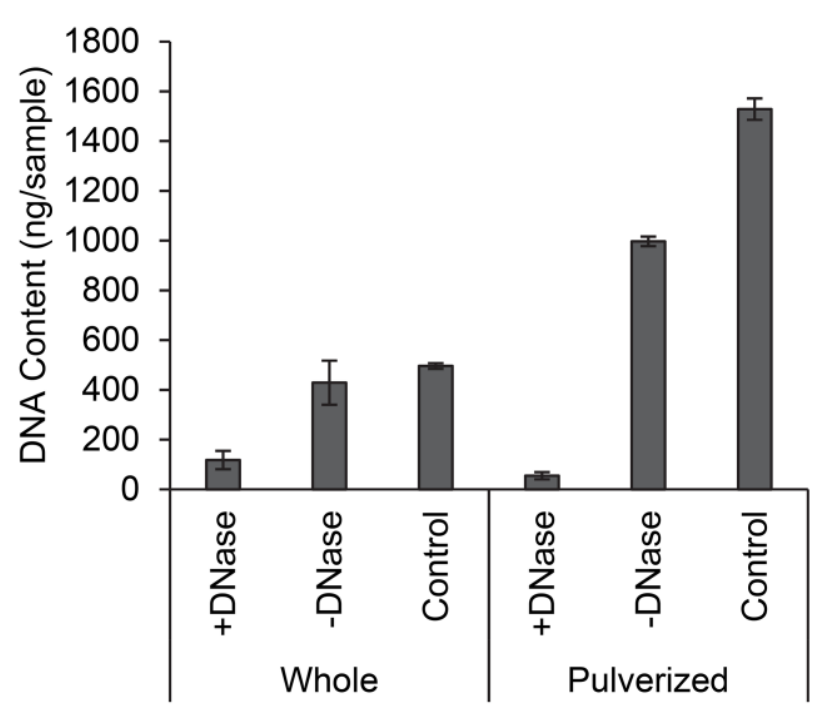

Figure S5. Effect of DNase treatment on whole and pulverized rice cells. Rice cell cultures grow as large aggregates. To determine if decellularization of cultured rice cells could be achieved with whole aggregates, an experiment was performed to quantify DNA content pre- and post-decellularization from whole and pulverized aggregates. For samples that should have identical DNA amounts, the DNA extraction from whole aggregates was significantly lower than from pulverized aggregates as evidenced by the higher DNA quantification from the pulverized aggregates. DNA removal was more effective from the pulverized aggregates (4\% versus $24 \%$, as calculated within the limitations of the extraction procedure). This data exemplifies the diffusion challenges to achieve adequate DNase penetration and DNA extraction for the whole aggregates. 


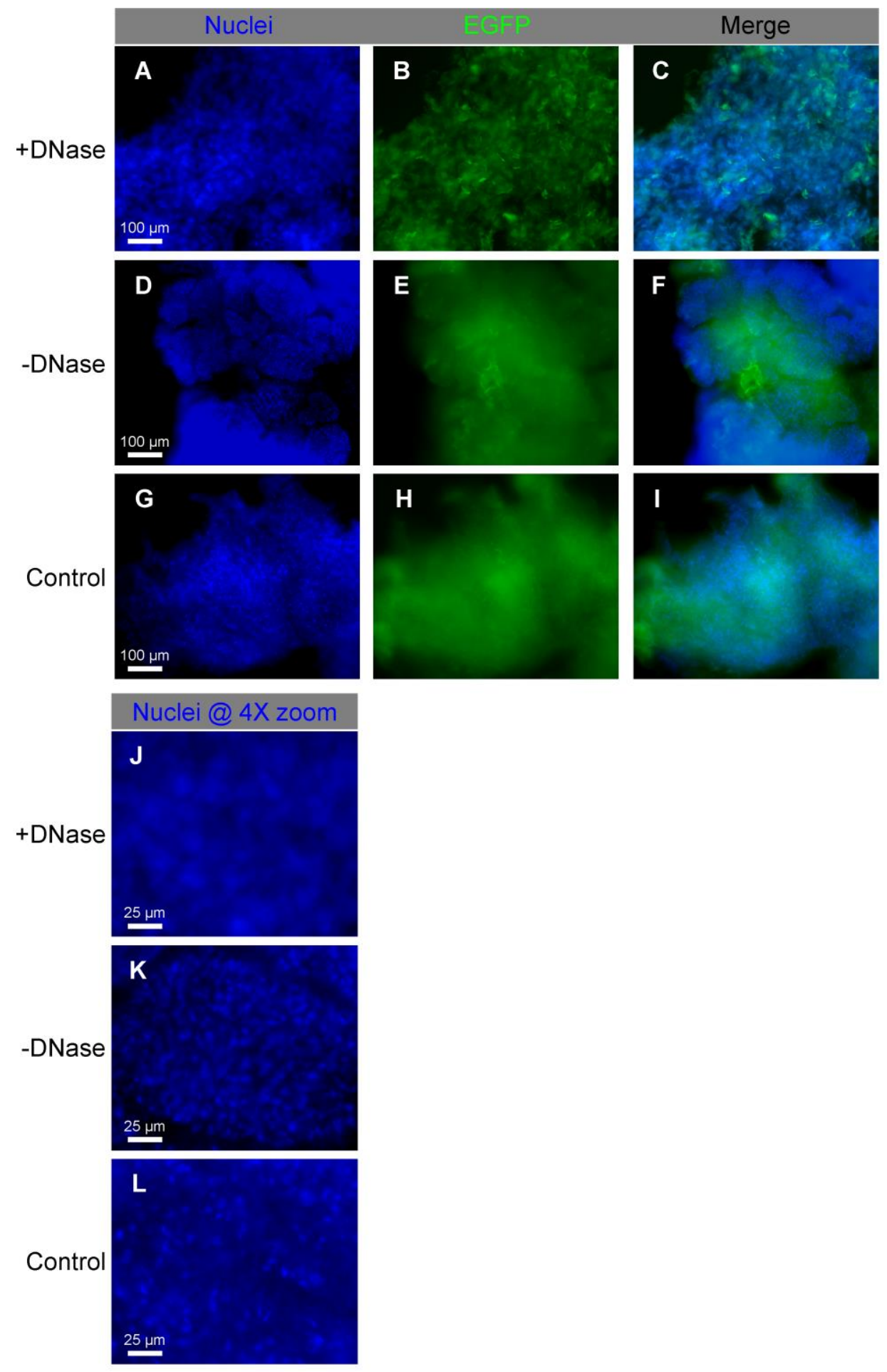

Figure S6. Fluorescent images of the processed rice cell-derived matrices. Images of pulverized rice cell-derived matrices after treatment with (A-C, J) $1 \mathrm{mg} / \mathrm{mL}$ DNase (+DNase) or (D-F, K) buffer-only (DNase) for $30 \mathrm{~min}$ at $37^{\circ} \mathrm{C}$. (G-I, L) Images of as-lyophilized BY-2 cell. (A, D, G) The nuclei of the + DNase treatment group were disrupted as evidenced by loss of focal staining as compared to the -DNase and Control groups. Autofluorescence by cellulose is also apparent in the blue channel. (B, E, H) Qualitatively, EGFP was retained within all matrices throughout the processing as evidenced by the observed fluorescence in the green channel. All fluorescent images in A-I were taken using the same magnification. 


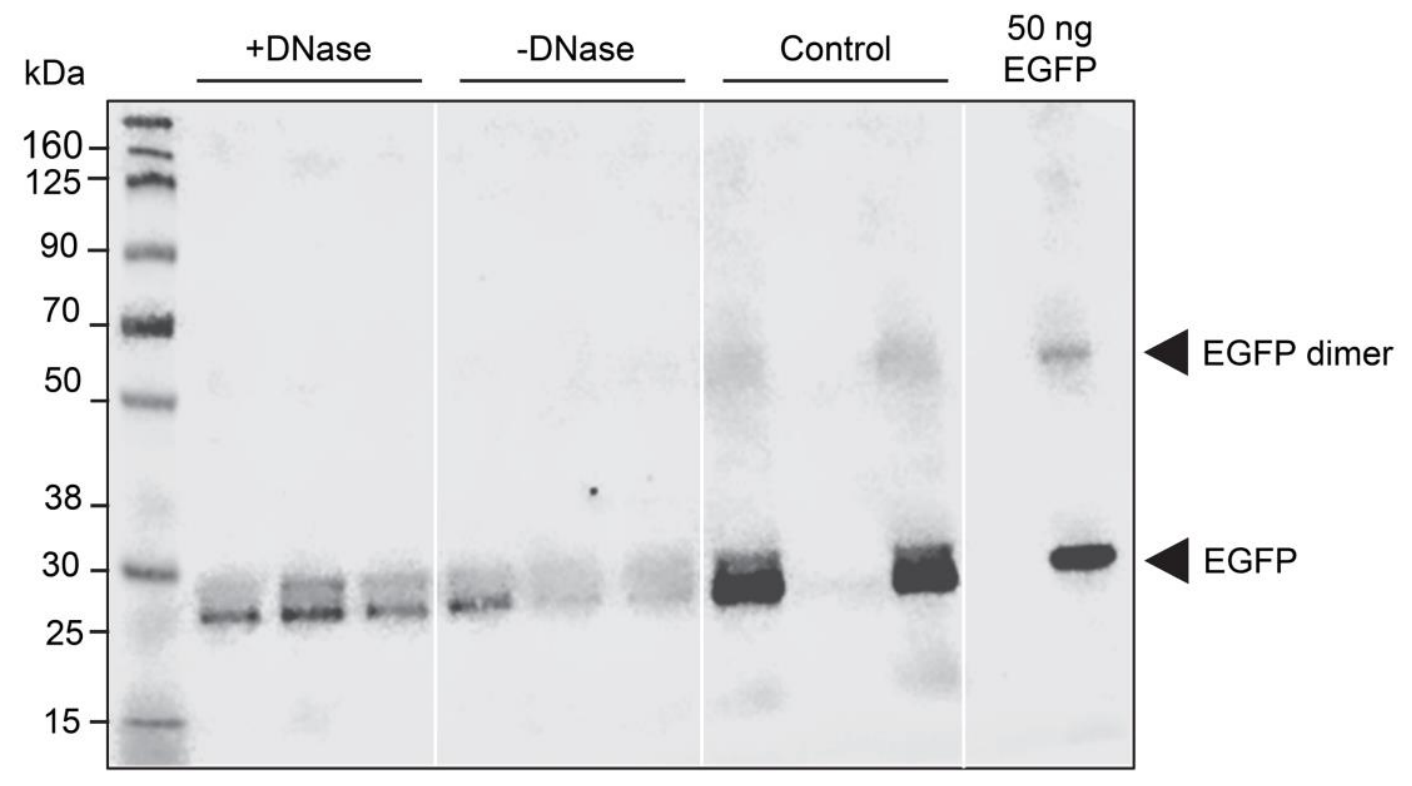

Figure S7. Western blot analysis of the processed rice cells. Lyophilized rice cells genetically engineered to express EGFP were treated with $1 \mathrm{mg} / \mathrm{mL}$ DNase (+DNase) or buffer-only (-DNase) for 30 min, washed, lyophilized, and processed for Western blotting. Unprocessed lyophilized rice cells (control) were used as an experimental control. Each lane is an independent sample. EGFP was retained within the matrices after processing (+DNase and -DNase: approximately $22 \%$ of the initial EGFP). 

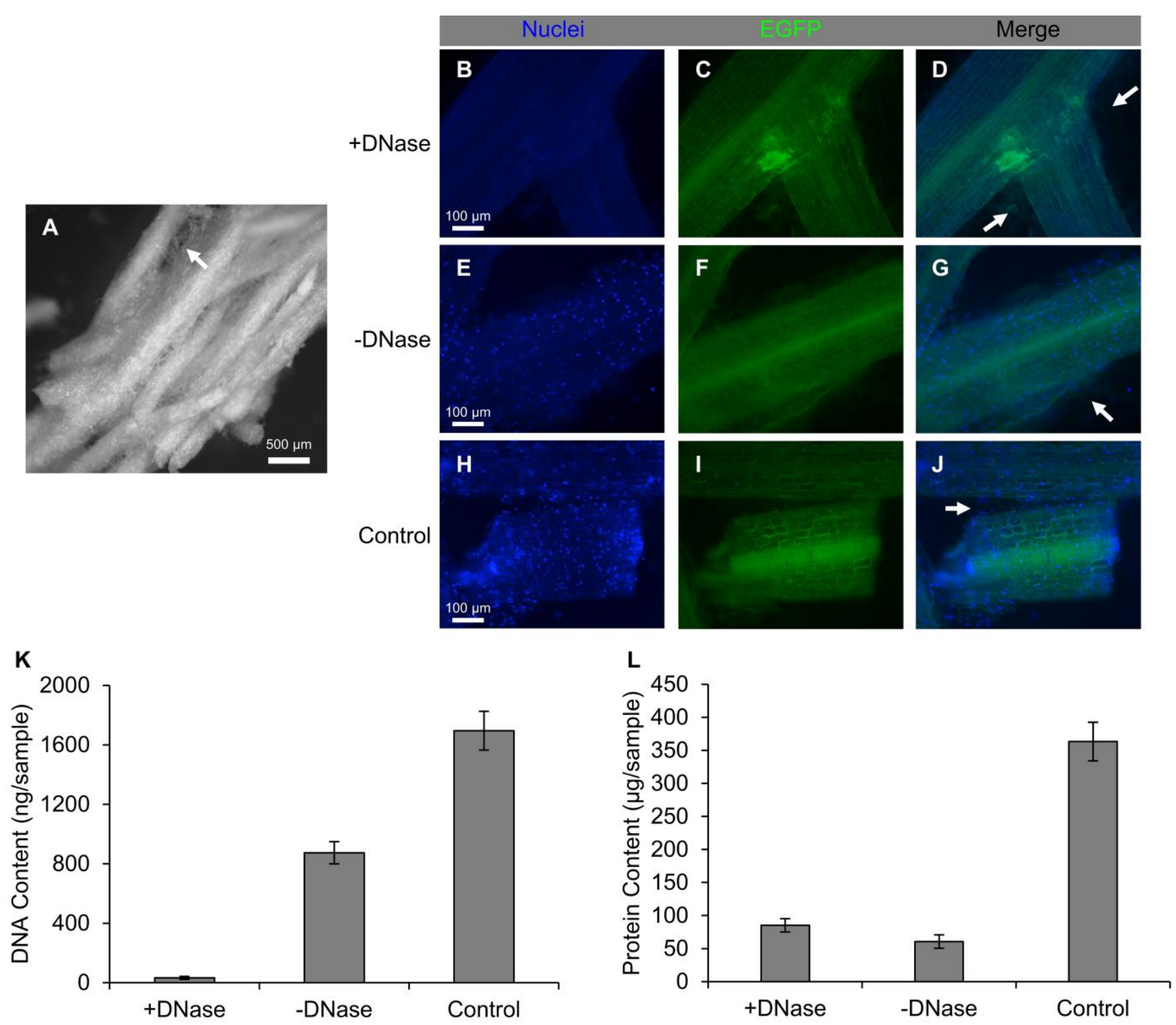

Figure S8. Characterization of processed hairy root matrices. (A) Stereoscope image of as-lyophilized hairy roots showing retention of root morphology. Fluorescent images of hairy root-derived matrices after treatment with (B-D) $1 \mathrm{mg} / \mathrm{mL}$ DNase (+DNase) or (E-G) buffer-only (-DNase) for $30 \mathrm{~min}$ at $37^{\circ} \mathrm{C}$. (HJ) Images of as-lyophilized hairy roots. $(\mathbf{B}, \mathbf{E}, \mathbf{H})$ The nuclei of the + DNase treatment group were disrupted as evidenced by loss of focal staining as compared to the -DNase treatment and Control group. (C, F, I) Qualitatively, EGFP was retained within all matrices throughout the processing as evidenced by the observed fluorescence in the green channel. For all images, arrows indicate areas of clear retention of "hairy" root morphology. (K) DNA content of samples treated with DNase (+DNase) or buffer-only (DNase) for $30 \mathrm{~min}$ at $37^{\circ} \mathrm{C}$ or as-lyophilized controls. (L) Protein content of samples treated with DNase (+DNase) or buffer-only (-DNase) for $30 \mathrm{~min}$ at $37^{\circ} \mathrm{C}$ or as-lyophilized controls. Data are presented as the mean \pm standard deviation of three samples. 


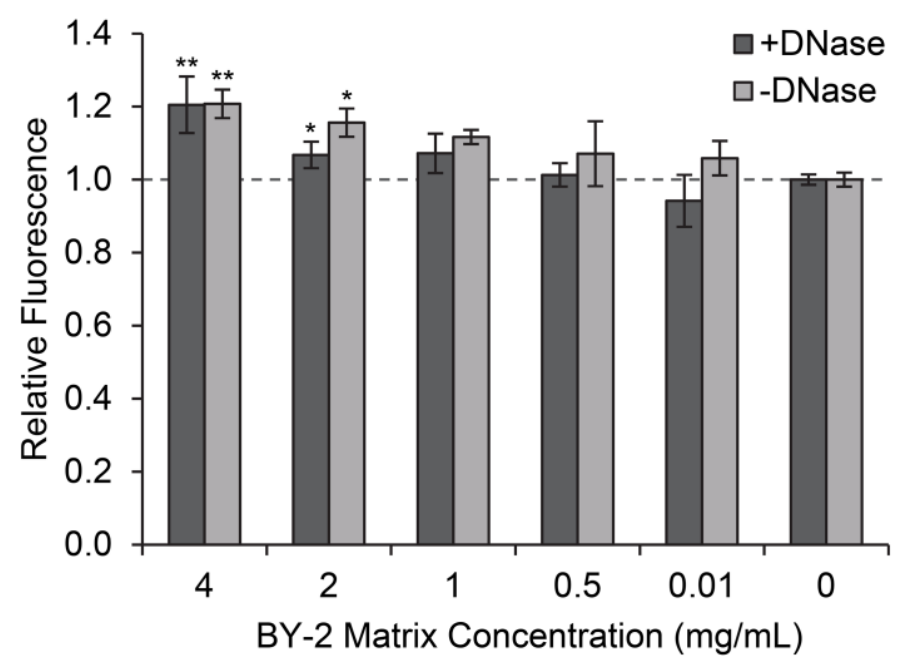

Figure S9. Monolayer cultured hFF response to BY-2 cell-derived matrices at varying concentrations. hFF metabolic activity, determined via resazurin metabolism, after treatment for 3 days with +DNase or -DNase BY-2 cell-derived matrices from $0.01 \mathrm{mg} / \mathrm{mL}$ to $4 \mathrm{mg} / \mathrm{mL}$. All data are normalized to untreated control cells $(0 \mathrm{mg} / \mathrm{mL})$. Data are presented as mean \pm standard deviation from three independent samples. Asterisks denote statistical difference from untreated control cells $(* \mathrm{p}<0.05$, $* * \mathrm{p}<0.01)$. 

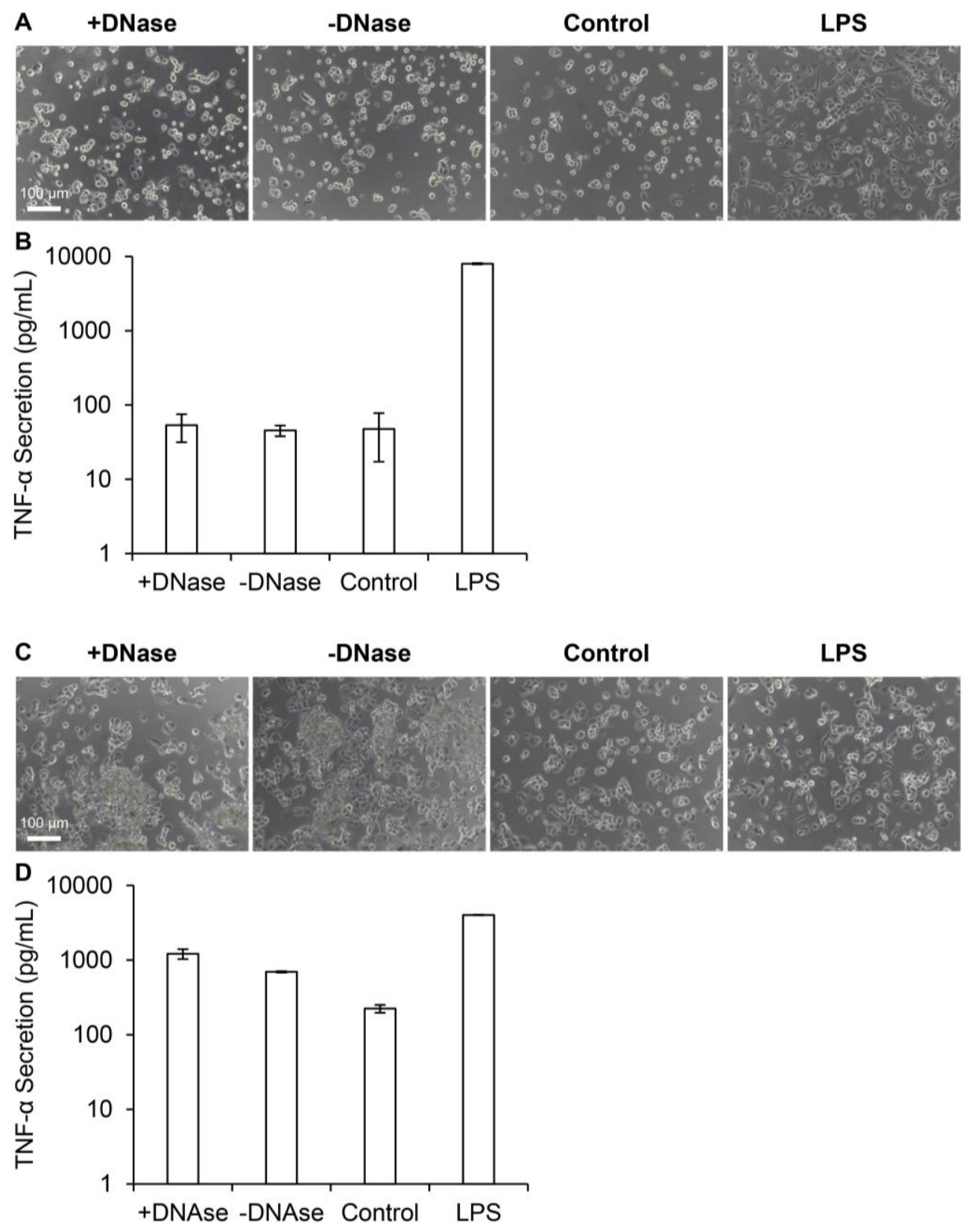

Figure S10. Effect of BY-2 cell-derived matrix conditioned medium and direct exposure to BY -2 cell-derived matrices on THP-1 cell-derived macrophages. TNF- $\alpha$ secretion by THP-1 cell-derived macrophages treated with (A) media with potential leachable components from the BY-2 cell-derived matrices (+DNase or -DNase) or (C) BY-2 cell-derived matrices directly (+DNase or -DNase). Controls were THP-1 cell-derived macrophages exposed to untreated medium (control, negative control) and medium containing LPS (LPS, positive control). (A) No differences in TNF- $\alpha$ secretion were observed between the +DNase, -DNase, and control groups. (C) Increased TNF- $\alpha$ secretion was observed when THP-1 cell-derived macrophages were directly treated with BY-2 cell-derived matrices. (A and C) All groups secreted levels of TNF- $\alpha$ that were less than the LPS group. Data are presented as mean \pm standard deviation from three samples. Phase contrast microscopy images of THP-1 cell-derived macrophages treated with (B) media with potential leachable components from the BY -2 cell-derived matrices $(+\mathrm{DNase}$ or -DNase) or (D) BY-2 cell-derived matrices directly (+DNase or -DNase). Differences between control and LPS group from one data set to the other are due to experimental variability as these experiments were performed separately. 